\title{
Análise faunística de gafanhotos Acridoidea da Volta Grande do Rio Xingu, área de influência direta da Hidrelétrica Belo Monte, Pará, Brasil
}

\author{
Faunistic analysis of grasshoppers Acridoidea in the Volta Grande of the Xingu River, area of \\ direct influence of the Belo Monte Hydroelectric Power Station, Pará, Brazil
}

\author{
Ana Lúcia Nunes-Gutjahr ${ }^{{ }^{*}}$ Carlos Elias de Souza Braga ${ }^{\mathrm{I}}$
}

\section{RESUMO}

O Rio Xingu possui um grande potencial hidrelétrico, especificamente na região da Volta da Grande, onde será instalada a Hidrelétrica de Belo Monte. Para a implementação desse empreendimento, foi necessário o estudo de impacto ambiental, considerando a fauna, flora e os aspectos sociais. Com o objetivo de realizar a análise faunistica das espécies de gafanhotos da Área de Influência Direta do Empreendimento Belo Monte, realizou-se um inventário, utilizando-se rede entomológica no periodo de novembro/2007 a agosto/2008. Foram coletados 2652 gafanhotos pertencentes a três famílias, 10 subfamílias, 18 tribos, 42 gêneros e 71 espécies. A análise faunistica evidenciou que $74 \%$ das espécies foram consideradas raras ou minimas e, quanto à constância, 54,9\% destas foram classificadas como acidentais. Os maiores índices de diversidade e de riqueza dos gafanhotos ocorreram na área A2, que será inundada para formar o lago da hidrelétrica, o que poderá causar extinção local dessas espécies.

Palavras-chave: Orthoptera, Inventário faunistico, Amazônia.

\section{ABSTRACT}

The Xingu River has a huge hydroelectric potential, specifically in the Volta Grande, where the hydroelectric power station of Belo Monte will be installed. For the implementation of this construction project an environmental impact study was necessary, considering the fauna, flora and the social aspects. In order to perform the faunistic analysis of grasshoppers species in the area of direct influence of the construction project of Belo Monte, an inventory was carried out using entomological nets from November/2007 to August/2008. It was collected 2652 grasshoppers, which belonged to 3 families, 10 subfamilies, 18 tribes, 42 genera and 71 species. The faunistic analysis demonstrated that $74 \%$ of the species were classified as rare or minimal and with regard to constancy $54.9 \%$ were classified as accidental. The highest levels of diversity and richness of grasshoppers occurred in the area A2 that will be flooded to form the hydroelectric lake, which may cause local extinction of these species.

Key words: Orthoptera, Faunistic inventory, Amazonia.

\section{INTRODUÇÃO}

O Rio Xingu é considerado um dos mais importantes rios da bacia hidrográfica amazônica. É classificado como rio de água clara pobre em sedimento, o que confere boa transparência às suas águas, podendo, entretanto, em alguns de seus trechos, ser rico em matéria dissolvida. Suas nascentes localizam-se no Planalto Central Brasileiro e até a sua foz percorre uma extensão de $1500 \mathrm{~km}$, com uma vazão de $7100 \mathrm{~m}^{3} \mathrm{~s}^{-1}$ (MIRANDA et al., 1988).

O Rio Xingu possui um trecho denominado Volta Grande do Xingu, onde o nível da água atinge até $70 \mathrm{~m}$, o que despertou interesse devido ao potencial hidráulico desse trecho. A área da Volta Grande do Xingu pertence a cinco municípios paraenses: Altamira, Anapu, Senador José Porfírio, Vitória do Xingu e Brasil Novo. Nessa área, foi aprovada a proposta para a implantação da hidrelétrica de Belo Monte, a qual é foco de intensa controvérsia, devido à magnitude e à natureza dos seus possíveis impactos ambientais e sociais (FEARNSIDE, 2005). Devido à intenção da implantação dessa hidrelétrica na região, estudos para a avaliação de impactos ambientais tiveram início em 1975 (FISHER, 1994). Mais

'Departamento de Ciências Naturais (DCNA), Centro de Ciências Sociais e Educação (CCSE), Universidade do Estado do Pará (UEPA), Rua Djalma Dutra s/n, 66113-010, Belém, PA, Brasil. E-mail: melcam@uol.com.br. *Autor para correspondência. 
recentemente, entre 2007 e 2009 , foram realizados estudos que abordaram a flora e a fauna da região. Entre a fauna pesquisada, os gafanhotos constituíram um dos grupos de invertebrados que foi inventariado.

Os gafanhotos são insetos pertencentes à ordem Orthoptera e possuem formas, hábitos e comportamentos diversos, destacando-se como um grupo de grande representatividade na região amazônica. Os Orthoptera possuem cerca de 26.000 espécies descritas, sendo destas 1.480 conhecidas para o Brasil e cerca 500 espécies para a Amazônia (NUNES-GUTJAHR \& BRAGA, 2012). Apresentam ampla distribuição mundial, não contendo registros de ocorrência apenas para a região Antártica (NUNESGUTJAHR \& BRAGA, 2011).

Os gafanhotos são herbívoros, mas algumas espécies podem também se alimentar de detritos vegetais e possuem importância ecológica e econômica. Sua importância ecológica baseia-se no fato de serem desfolhadores naturais das florestas e ocuparem, ainda, outros nichos consideráveis como comedores de matéria orgânica vegetal em decomposição, musgos, entre outros. Dessa forma, são importantes na reciclagem da matéria vegetal. Também, destacam-se por fazerem parte da dieta alimentar de muitos vertebrados, tais como aves, anfíbios e mamíferos, sendo, por isso, considerados elementos indispensáveis na cadeia trófica (NUNESGUTJAHR \& BRAGA, 2011). Vale ressaltar que os gafanhotos possuem íntima relação com a vegetação que lhes serve de substrato e alimentação e, por isso, a composição de suas espécies é importante para indicar a qualidade ambiental de determinada região, reforçando que esses insetos são importantes bioindicadores ecológicos. A importância econômica dos gafanhotos decorre dos danos que algumas espécies podem causar em áreas cultivadas, as quais podem destruir em curto espaço de tempo plantações inteiras (NUNES-GUTJAHR \& BRAGA, 2011). Outra forma de prejuízo é observada quando gradativamente danificam os tecidos vegetais com suas oviposições endofíticas, que são evidenciadas, principalmente, nas espécies semiaquáticas (NUNES et al., 2005).

Considerando que SILVEIRA NETO et al. (1995) admite que estudos de análise faunística permitem a avaliação de impacto ambiental, tendo por base as espécies de insetos como indicadores ecológicos, este trabalho tem como objetivo analisar a composição, abundância, frequência, diversidade e riqueza de espécies da comunidade de gafanhotos Acridoidea na Área de Influência Direta (AID) do Empreendimento Belo Monte. Ressalta-se ainda que se trata de um trabalho pioneiro e relevante, por se desenvolver em uma área que sofrerá grandes alterações ambientais, devido à implantação de uma usina hidrelétrica e que irá contribuir para subsidiar estudos futuros na região, os quais comparem a fauna de Acridoidea prévia e posterior ao impacto ocasionado pela construção da represa, além de ampliar o conhecimento sobre a biodiversidade amazônica.

\section{MATERIAL E MÉTODOS}

Área de estudo

O estudo foi realizado na Área de Influência

Direta (AID) do empreendimento Belo Monte na região da Volta Grande do Rio Xingu, que abrangeu os polígonos fitofisionômicos previamente determinadas como A1 (A1MD - floresta ombrófila aberta com cipó e palmeira latifoliada em relevo acidentado; A1IG ilha fluvial de floresta ombrófila aluvial com dossel contínuo periodicamente alagada; A1ME - floresta ombrófila aberta com palmeira latifoliada em relevo acidentado), A2 (todas as áreas em floresta ombrófila aberta com cipó e palmeira latifoliada em relevo acidentado) e A3 (A3ME - floresta ombrófila aberta com palmeira latifoliada com relevo acidentado; A3IBV - ilha fluvial de floresta ombrófila aluvial com dossel contínuo periodicamente alagada; A3MD - floresta ombrófila densa latifoliada com dossel emergente) (Figura 1).

Os gafanhotos foram coletados em ambiente de floresta nas trilhas do inventário de fauna dos estudos prévios para a implantação da hidrelétrica, conforme a seguir: Área A1: região de Salvaterra (Altamira): A1MD ou Itapuama (0336'17'S; 52 20'30' W); A1IG ou Ilha Grande (03'36'14'"S; 52 22'24'”W); A1ME ou Agropecuária WR (03³1'37'S; 52²2'25'W). Área A2: Vitória do Xingu: A2T50 ou Travessão 50 $\left(03^{\circ} 12^{\prime} 12^{\prime}\right.$ S; 51 $\left.48^{\circ} 17^{\prime} \mathrm{W}\right)$; A2IM ou Igarapé de Maria (03⒉ '53"'S; 51 $\left.{ }^{\circ} 54^{\prime} 40^{\prime \prime} \mathrm{W}\right)$; A2IG ou Igarapé Galhoso (0320'26"S; 51 $\left.{ }^{\circ} 56^{\circ} 48^{\prime \prime} \mathrm{W}\right)$. Área A3: Volta Grande-Jericoá: A3ME ou Bom Jardim (Vitória do Xingu) (03'24'47'S; 51 $\left.45^{\circ} 22^{\prime \prime} \mathrm{W}\right)$; A3IBV ou Ilha Bela Vista (Anapu) $\left(03^{\circ} 24^{\prime} 03^{\prime \prime} \mathrm{S} ; 51^{\circ} 43^{\prime} 25^{\prime \prime} \mathrm{W}\right)$; A3MD ou Barra do Vento (Anapu) (03⒉ $35^{\prime \prime} \mathrm{S}$; $\left.51^{\circ} 39^{\prime} 47^{\prime \prime} \mathrm{W}\right)$.

\section{Método de coleta}

As coletas foram realizadas nos meses de novembro/2007, fevereiro, abril e agosto/2008, seguindo protocolo estabelecido pelo estudo de impacto ambiental do empreendimento Belo 


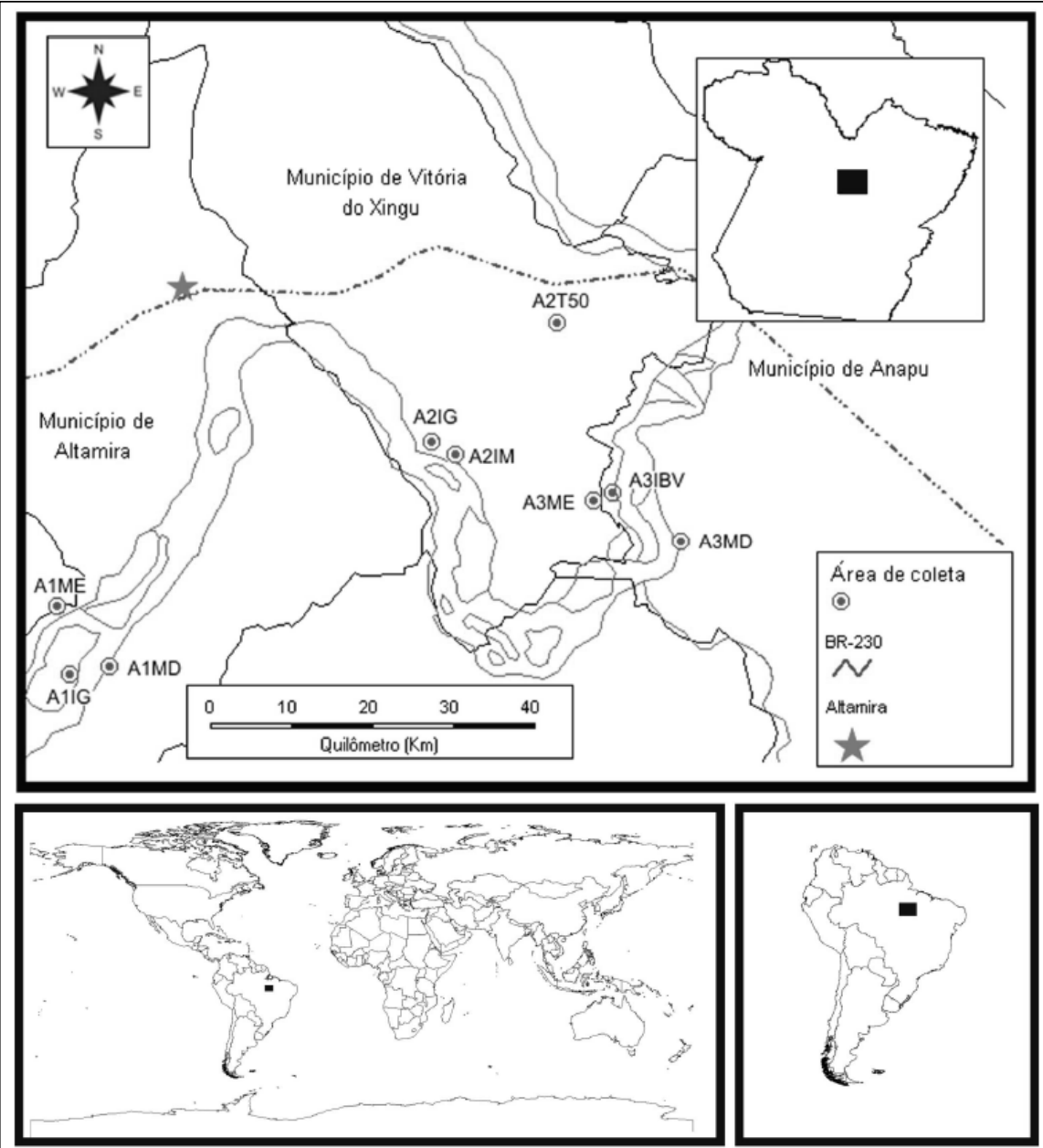

Figura 1 - Mapeamento das localidades de amostragem e pontos de coletas de gafanhotos Acridoidea na Volta Grande do Rio Xingu, Pará.

Monte. A coleta dos gafanhotos Acridoidea foi baseada em captura ativa (RAFAEL, 2002) com rede entomológica (aro de $40 \mathrm{~cm}$ de diâmetro, saco coletor de $60 \mathrm{~cm}$ de comprimento e cabo de madeira de $100 \mathrm{~cm}$ comprimento). O tempo de captura foi de 180 minutos em cada trilha, iniciando-se às oito horas da manhã. A captura dos gafanhotos foi realizada por quatro coletores treinados e experientes neste método de coleta, que caminhavam distando entre si cerca de $5 \mathrm{~m}$ nas áreas de amostragem. Todos os gafanhotos capturados foram transportados para o laboratório para tratamento (triagem, montagem e etiquetagem). Cada trilha amostrada foi georreferênciada com GPS (e-Trex). O material coletado foi tratado, identificado e incorporado ao acervo da Coleção de Invertebrados do Museu Paraense Emílio Goeldi (MPEG). Para a identificação dos gafanhotos em espécie e morfoespécie, utilizou-se estereomicroscópio, com o auxílio de chaves dicotômicas de literatura pertinente e por comparação com espécimes identificados da Coleção do MPEG.

Análise faunística

Para a caracterização da comunidade de gafanhotos, foram avaliadas as medidas faunísticas frequência relativa (FR), abundância (A) e Constância (C). O parâmetro faunístico frequência relativa (FR) foi determinado através da porcentagem de indivíduos de cada espécie em relação ao total de gafanhotos obtidos nas coletas, podendo as espécies serem classificadas como muito frequente $(\mathrm{FR}>5 \%)$, frequente $(\mathrm{FR}=5 \%)$ e pouco frequente $(\mathrm{FR}<5 \%)$ (THOMAZINI \& THOMAZINI, 2002). Para a abundância (A), utilizaram-se as medidas segundo AMĖDÉGNATO \& DESCAMPS (1978), com base na frequência relativa (FR) das espécies, 
classificando-as em cinco categorias faunísticas: dominante (FR $\geq 5 \%$ ), abundante ( $F R \geq 2,5 \%,<5 \%$ ), comum $(\mathrm{FR} \geq 1,5 \%,<2,5 \%)$, rara $(\mathrm{FR}>0,5 \%,<1,5 \%)$ e mínima $(\mathrm{FR}<0,5 \%)$. A medida faunística de constância (C) seguiu-se SILVEIRA NETO et al. (1976), que considerou a percentagem de coletas que continham uma determinada espécie, classificadas em constante $(\mathrm{C}>50 \%)$, acessória $(\mathrm{C}>25 \%,<50 \%)$ e acidental $(\mathrm{C}<25 \%)$ (THOMAZINI \& THOMAZINI, 2002). Também, considerou-se o índice de diversidade de Shanon-Wiener $(\mathrm{H})$, determinado pelo programa DivEs 2.0 (RODRIGUES, 2005) e a riqueza de espécies para cada área.

\section{RESULTADOS E DISCUSSÃO}

Durante o período de amostragem, foram coletados 2652 gafanhotos (Acridoidea) pertencentes a três famílias, 10 subfamílias, 18 tribos, 42 gêneros e 71 espécies (Tabela 1). A família Acrididae foi a mais abundante com 1726 espécimes de gafanhotos amostrados (65,1\%). A família Pyrgomorphidae teve a menor abundância com apenas 8 espécimes capturados (0,3\%), enquanto que Romaleidae representou 34,6\%. A maior representatividade de Acrididae pode ser explicada por se tratar da família mais especiosa e também que possui as espécies de gafanhotos mais comumente encontradas em ambientes naturais, como já foi observado em outros estudos realizados no Cerrado (GUERRA et al., 2012), na Amazônia (NUNES-GUTJAHR \& BRAGA, 2010) e em áreas de fragmentos florestais e de cultivares na Flona de Chapecó, em Santa Catarina (LUTINSKI et al., 2011).

As espécies Amblytropidia sp., Episomacris sp., Locheuma brunneri, Silvitettix sp.1, Colpolopha obsoleta, Colpolopha sp. e Epiprora hilaris foram classificadas como muito frequentes, dominantes e constantes (Tabela 1). Ressalta-se que a classificação faunística de gafanhotos pode variar de acordo com a região geográfica. $\mathrm{O}$ trabalho de GRACIANI et al. (2005), em uma floresta subtropical do alto Uruguai, onde não foi constatada a existência de espécies muito frequentes, reforça que em ambientes distintos a comunidade de gafanhotos pode apresentar diferentes composição e frequência de espécies.

Neste estudo, entre as 71 espécies encontradas, quatro já haviam sido analisadas em inventários faunísticos de outras regiões do Brasil, onde foram classificadas de forma distinta: Amblytropidia sp., aqui considerada como muito frequente, dominante e constante (Tabela 1), foi classificada por GRACIANI et al. (2005) e por LUTINSKI et al. (2011) como pouco frequente, rara e acidental; Abracris flavolineata (pouco frequente, rara e constante: Tabela 1) também pelos mesmos autores, foi classificada como pouco frequente, rara e acidental; Orphulella punctata (pouco frequente, rara e constante: Tabela 1) apresentou a mesma classificação por GRACIANI et al. (2005), entretanto, na classificação de LUTINSKI et al. (2011) ela expressou uma única diferença quanto a ser uma espécie acidental; Metaleptea adspersa (pouco frequente, mínima e acidental: Tabela 1) para GRACIANI et al. (2005), mostrou-se pouco frequente, rara e acidental e, por LUTINSKI et al. (2011), como muito frequente, dominante e acessória. Isso indica que, de acordo com determinado ambiente e bioma ao qual pertencem, as espécies de gafanhotos podem apresentar outros padrões faunísticos. Vale ressaltar que as espécies consideradas predominantes foram aquelas que se destacaram por obter os maiores índices faunísticos de abundância, frequência, constância e dominância (SILVEIRA NETO et al., 1995). No entanto, durante o estudo, apenas Locheuma brunneri apresentou Constância de $100 \%$, indicando que esta espécie ocorreu em todas as áreas amostradas. Esse resultado, possivelmente, é decorrente por se tratar de um gafanhoto da subfamília Ommatolampinae, a qual reúne espécies que exploram ambientes de subbosque com vegetação baixa (NUNES-GUTJAHR \& BRAGA, 2011) e diversificada, que se encontra em ambientes de mata alta, com certa luminosidade, como áreas próximas às bordas das florestas. Ressalta-se que esta espécie é pouco estudada, porém é conhecida sua ampla distribuição geográfica para a região amazônica (AMÉDÉGNATO, 1997).

A análise da composição de espécies da Volta Grande do Rio Xingu mostrou que, nas áreas A1, A2 e A3 da AID da hidrelétrica Belo Monte, existem oito espécies de gafanhotos dominantes, equivalentes a $63,8 \%$ dos espécimes coletados e $11,3 \%$ das espécies da área estudada; quatro espécies são abundantes $(11,1 \%$ de espécimes e $5,6 \%$ das espécies); seis espécies comuns (11,2\% de espécimes e $8,5 \%$ das espécies); 12 espécies raras $(9,0 \%$ de espécimes e $16,9 \%$ das espécies $)$ e 41 espécies mínimas $(4,9 \%$ de espécimes e $57,7 \%$ das espécies). Além disso, 24 espécies foram consideradas constantes, oito acessórias e 39 acidentais. Também, constatou-se que oito espécies eram muito frequentes e 63 pouco frequentes, não sendo nenhuma espécie classificada como frequente, resultado este que também já foi evidenciado nos estudos de GRACIANI et al. (2005) (Tabela 1). 
Tabela 1 - Famílias, espécies, número de exemplares e classificação em categorias faunísticas de gafanhotos inventariados na Volta Grande do Rio Xingu, no período de novembro de 2007 a agosto de 2008.

\begin{tabular}{|c|c|c|c|c|c|c|c|c|c|c|c|}
\hline Família & Espécie & $\mathrm{NE}$ & FR & A & $\mathrm{C}$ & Família & Espécie & $\mathrm{NE}$ & FR & A & $\mathrm{C}$ \\
\hline \multirow[t]{36}{*}{ Acrid } & Abracris dilecta & 32 & $\mathrm{PF}$ & $\mathrm{R}$ & $\mathrm{Co}$ & Acrid & Poecilocloeus sp.7 & 2 & $\mathrm{PF}$ & M & Ac \\
\hline & Abracris flavolineata & 78 & $\mathrm{PF}$ & $\mathrm{AB}$ & $\mathrm{Co}$ & & Poecilocloeus sp.8 & 2 & $\mathrm{PF}$ & M & Ac \\
\hline & Amblytropidia sp. & 192 & MF & $\mathrm{D}$ & $\mathrm{Co}$ & & Poecilocloeus sp.9 & 2 & $\mathrm{PF}$ & M & Ac \\
\hline & Anablysis teres & 22 & $\mathrm{PF}$ & $\mathrm{R}$ & Ac & & Psiloscirtus olivaceus & 48 & $\mathrm{PF}$ & $\mathrm{CO}$ & Co \\
\hline & Chloropseustes flavipes & 16 & $\mathrm{PF}$ & $\mathrm{R}$ & Ac & & Psiloscirtus sp. & 2 & $\mathrm{PF}$ & M & Ac \\
\hline & Chloropseustes leucotylus & 50 & $\mathrm{PF}$ & $\mathrm{CO}$ & $\mathrm{Co}$ & & Silvitettix sp.1 & 140 & MF & $\mathrm{D}$ & $\mathrm{Co}$ \\
\hline & Clematodina eckardtiana & 134 & MF & $\mathrm{D}$ & $\mathrm{Co}$ & & Silvitettix sp. 2 & 49 & $\mathrm{PF}$ & $\mathrm{CO}$ & Co \\
\hline & Compsacris pulcher & 81 & $\mathrm{PF}$ & $\mathrm{AB}$ & $\mathrm{Co}$ & & Sitalces sp.1 & 65 & $\mathrm{PF}$ & $\mathrm{CO}$ & Co \\
\hline & Copiocera prasin & 4 & $\mathrm{PF}$ & M & Ac & & Sitalces $\mathrm{sp} .2$ & 7 & $\mathrm{PF}$ & M & Ace \\
\hline & Copiocera sp. & 1 & $\mathrm{PF}$ & M & Ac & & Sitalces sp. 3 & 1 & $\mathrm{PF}$ & $\mathrm{M}$ & Ac \\
\hline & Copiocera surinamensis & 22 & $\mathrm{PF}$ & $\mathrm{R}$ & $\mathrm{Co}$ & & Stenopola dorsalis & 7 & $\mathrm{PF}$ & $\mathrm{M}$ & $\mathrm{Co}$ \\
\hline & Cornops frenatum & 48 & $\mathrm{PF}$ & $\mathrm{CO}$ & Co & & Stenopola sp. & 1 & $\mathrm{PF}$ & M & Ac \\
\hline & Coscineuta pulchripes & 28 & $\mathrm{PF}$ & $\mathrm{R}$ & Ace & & Syntomacris sp.1 & 4 & $\mathrm{PF}$ & M & Ac \\
\hline & Cylindrotettix attenuatus & 3 & $\mathrm{PF}$ & M & Ac & & Syntomacris sp.2 & 12 & $\mathrm{PF}$ & $\mathrm{R}$ & $\mathrm{Co}$ \\
\hline & Episomacris sp. & 187 & MF & $\mathrm{D}$ & $\mathrm{Co}$ & & Syntomacris sp. 3 & 10 & $\mathrm{PF}$ & M & Ace \\
\hline & Eumastusia koebelei & 4 & $\mathrm{PF}$ & M & Ac & & Syntomacris sp.4 & 1 & $\mathrm{PF}$ & M & Ac \\
\hline & Locheuma brunneri & 267 & MF & $\mathrm{D}$ & $\mathrm{Co}$ & & Syntomacris virgata & 1 & $\mathrm{PF}$ & M & $\mathrm{Ac}$ \\
\hline & Lysacris sp. & 2 & $\mathrm{PF}$ & M & $\mathrm{Ac}$ & & Tetrataenia surinama & 4 & $\mathrm{PF}$ & $\mathrm{M}$ & $\mathrm{Ac}$ \\
\hline & Metaleptea adspersa & 2 & $\mathrm{PF}$ & M & Ac & & Vilerna aeneooculata & 49 & $\mathrm{PF}$ & $\mathrm{CO}$ & $\mathrm{Co}$ \\
\hline & Monachidium lunum & 2 & $\mathrm{PF}$ & M & Ac & & Xenismacris aetoma & 2 & $\mathrm{PF}$ & $\mathrm{M}$ & Ac \\
\hline & Omalotettix sp. & 4 & $\mathrm{PF}$ & M & $\mathrm{Ac}$ & & & & & & \\
\hline & Orphula sp. & 1 & $\mathrm{PF}$ & M & Ac & Pyrgom & Omura congrua & 8 & $\mathrm{PF}$ & M & Ac \\
\hline & Orphulella concinnula & 18 & $\mathrm{PF}$ & $\mathrm{R}$ & Co & Romale & Adrolampis sp. & 1 & $\mathrm{PF}$ & M & Ac \\
\hline & Orphulella punctata & 20 & $\mathrm{PF}$ & $\mathrm{R}$ & Co & & Colpolopha obsoleta & 297 & MF & $\mathrm{D}$ & Co \\
\hline & Orphulella sp.1 & 1 & $\mathrm{PF}$ & M & $\mathrm{Ac}$ & & Colpolopha sp. & 298 & MF & $\mathrm{D}$ & $\mathrm{Co}$ \\
\hline & Orphulella $\mathrm{sp} .2$ & 2 & $\mathrm{PF}$ & M & Ac & & Epiprora hilaris & 177 & MF & $\mathrm{D}$ & Co \\
\hline & Orphulella sp. 3 & 1 & $\mathrm{PF}$ & $\mathrm{M}$ & Ac & & Habrolampis sp. & 1 & $\mathrm{PF}$ & M & Ac \\
\hline & Paropaon laevifrons & 53 & $\mathrm{PF}$ & $\mathrm{CO}$ & Ac & & Helolampis militaris & 1 & $\mathrm{PF}$ & $\mathrm{M}$ & Ac \\
\hline & Poecilocloeus sp.1 & 3 & $\mathrm{PF}$ & M & Ac & & $\begin{array}{l}\text { Maculiparia obtusa } \\
\text { solimoensis }\end{array}$ & 17 & $\mathrm{PF}$ & $\mathrm{R}$ & Ace \\
\hline & Poecilocloeus sp.10 & 1 & $\mathrm{PF}$ & M & Ac & & Ophthalmolampis sp.1 & 1 & $\mathrm{PF}$ & M & Ac \\
\hline & Poecilocloeus sp.11 & 1 & $\mathrm{PF}$ & M & Ac & & Ophthalmolampis sp.2 & 2 & $\mathrm{PF}$ & $\mathrm{M}$ & Ac \\
\hline & Poecilocloeus sp.2 & 12 & $\mathrm{PF}$ & $\mathrm{R}$ & Ace & & $\begin{array}{l}\text { Phaeoparia lineaalba } \\
\text { lineaalba }\end{array}$ & 71 & $\mathrm{PF}$ & $\mathrm{AB}$ & Co \\
\hline & Poecilocloeus sp. 3 & 9 & $\mathrm{PF}$ & M & Ace & & Prionacris sp. & 3 & $\mathrm{PF}$ & M & Ac \\
\hline & Poecilocloeus sp.4 & 4 & $\mathrm{PF}$ & M & Ace & & Prionolopha serrata & 24 & $\mathrm{PF}$ & $\mathrm{R}$ & $\mathrm{Co}$ \\
\hline & Poecilocloeus sp.5 & 1 & $\mathrm{PF}$ & M & Ac & & Pseudonautia sp. & 9 & $\mathrm{PF}$ & M & Ac \\
\hline & Poecilocloeus sp.6 & 11 & $\mathrm{PF}$ & M & Ace & & Tropidacris collaris & 16 & $\mathrm{PF}$ & $\mathrm{R}$ & Ac \\
\hline Total & & & & & & & 2652 & & & & \\
\hline
\end{tabular}

Acrid $=$ Acrididae $;$ Pyrgom $=$ Pyrgomorphidae $;$ Romale $=$ Romaleidae $;$ NE $=$ Número de Exemplares; FR $=$ Frequência $;$ PF: Pouco Frequente; $\mathrm{MF}=$ Muito Frequente; $\mathrm{A}=$ Abundância; $\mathrm{D}=$ Dominante; $\mathrm{AB}=$ Abundante; $\mathrm{CO}=\mathrm{Comum} ; \mathrm{R}=\mathrm{Rara} ; \mathrm{M}=\mathrm{Mínima} ; \mathrm{C}=$ Constância; $\mathrm{Co}=$ Constante; $\mathrm{Ac}=$ Acidental; Ace $=$ Acessória .

Através dessa análise, pode-se afirmar que, das 71 espécies de gafanhotos coletadas, neste estudo, $74,6 \%$ estão classificadas como raras e mínimas, o que serve de indicativo para a valoração das áreas estudadas, pois reúnem um grande número de espécies de gafanhotos dessas categorias faunísticas (Tabela 1). Quanto a isso, é sabido que áreas detentoras de grande número de espécies raras podem ser consideradas mais vulneráveis à extinção, visto que, por possuírem baixa densidade, são suscetíveis ao isolamento reprodutivo (LYONS et al., 2005). Ressalta-se, ainda, a importância da realização de estudos que identifiquem o papel dessas espécies nos ambientes, também, servem para indicar que ações 
causadoras de impacto ambiental, a serem implantadas nessas áreas, devem considerar tais informações.

Quanto ao índice de diversidade $(\mathrm{H})$, os resultados mostraram que a área denominada Ilha Grande (A1IG) teve o menor valor (Ds: 0.4397), com riqueza de apenas 11 espécies amostradas. Entre estas, destaca-se Epriprora hilaris, com representatividade de $73,75 \%$ de todos os espécimes capturados nessa área. Esse resultado pode ser decorrente do fato de que a Ilha Grande fica completamente inundada no período de cheia do Rio Xingu, sendo, por isso, caracterizada por uma vegetação adaptada a essa dinâmica hídrica, com árvores de porte baixo e sub-bosque uniforme, o que poderia estar restringindo as opções de alimentação para outras espécies de gafanhotos e consequentemente a diversidade destes. Quanto a isso, DESCAMPS (1978) admite a existência de especificidade de gafanhotos em função da vegetação dos ambientes, a qual eles utilizam como alimento e substrato. Pelo exposto, pode-se admitir que a vegetação da A1IG constitui o recurso alimentar e de substrato utilizado por poucas espécies de gafanhotos, sendo mais bem aproveitada por $\boldsymbol{E}$. hilaris.

Apesar da Ilha Grande apresentar um baixo índice de diversidade, possui um papel importante na região quanto à manutenção das espécies locais, tendo vista que a espécie Anablysis teres teve ocorrência somente nesta área e na área A3MD. Quanto a isso, $\boldsymbol{A}$. teres na A1IG teve representatividade de 90,9\% dos espécimes inventariados no estudo e na área A3MD de $9,1 \%$, sendo que esta espécie não foi encontrada em nenhuma das outras sete áreas estudadas. Deve-se destacar, ainda, que Prionacris sp. também ocorreu apenas nessa área e na Ilha Bela Vista (A3IBV), o que reforça o papel da Ilha Grande na sustentação da comunidade de gafanhotos na região da volta grande do Xingu. Pelo exposto, SERAFINI et al. (2010) consideram que as ilhas são ambientes particulares, pois, devido ao seu isolamento geográfico, geralmente abrigam uma biodiversidade peculiar e com grande número de espécies endêmicas. Vale ressaltar que, em geral, a biodiversidade de ilhas é bastante particular e, por mais que estes ambientes representem somente $5 \%$ da cobertura terrestre do planeta, cerca de um terço de todas as espécies de mamíferos, aves e anfíbios ameaçadas se encontram nestes locais (FONSECA et al., 2006).

Entre as áreas estudadas, as que apresentaram maior índice de diversidade e de riqueza foram: A2IG (Ds: 0.914; Riqueza: 33 espécies), A2IM (Ds: 0.9146; Riqueza: 31 espécies) e A2T50 (Ds: 0.9224; Riqueza: 30 espécies). Também foram registradas nestas áreas endemismos para algumas espécies: Poecilocloeus sp.7 endêmico da área A2IG; Lysacris sp., Poecilocloeus sp. 9, Poecilocloeus sp.10 e Syntomacris virgata endêmicos da área A2IM e Eumastusia koebelei, Orphula sp., Poecilocloeus sp.1, Poecilocloeus sp.11 e Syntomacris sp.4 endêmicos da área A2T50. Existem outras quatro espécies que são endêmicas de mais de uma das áreas citadas: Chloropseustes flavipes, Copiocera lepida (A2IG e A2IM); Monachidium lunum (A2IM e A2T50); Poecilocloeus sp.2 (A2IG, A2IM e A2T50). Para as áreas mencionadas anteriormente, ressalta-se que sua importância não se limita apenas em apresentar maior riqueza de espécies, mas por reter um maior grau de endemismo na Volta Grande do Rio Xingu. Esse resultado, de que tais áreas possuem 14 espécies endêmicas de gafanhotos para essa porção da região Amazônica, é motivo de preocupação, quanto à conservação dessas espécies. Isso se deve ao fato de que as áreas A2IG, A2IM e A2T50 do complexo Belo Monte, serão as mais afetadas pela instalação da hidrelétrica, pois, neste local, será formado o lago (reservatório) que alimentará a casa de força do empreendimento. Portanto, com a formação do lago, ocorrerá à inundação das referidas áreas, que possivelmente ocasionará a extinção local de tais espécies.

Reforçando as afirmações tecidas anteriormente, pode-se dizer que 53 espécies $(74,6 \%)$ das 71 espécies inventariadas na Volta Grande do Rio Xingu, se encontram nas áreas A2IG, A2IM e A2T50, demonstrando que os locais de maior riqueza específica e que reúne a maior concentração de espécies endêmicas para a área do complexo Belo Monte serão severamente prejudicadas pela implementação da usina hidrelétrica. Entretanto, é sabido que a extinção de uma única espécie, em dado local, ocasiona desequilíbrio ecossistêmico, tendo em vista que espécies que mantêm relações ecológicas com esta, também serão afetadas e consequentemente extintas. Quanto às espécies de gafanhotos da região da bacia do Rio Xingu, ressalta-se que poucas delas possuem informações biológicas, ou seja, poucas foram estudadas, não havendo, portanto conhecimento sobre a história natural de tais espécies e muito menos sobre os papéis ecológicos que desempenham nos ecossistemas onde vivem. Para gafanhotos, esses estudos são fundamentais, visto que se tratam de insetos que possuem espécies pragas de plantas cultivadas. Dessa forma, o desconhecimento de tais espécies desperta preocupação para a região 
de estudo, pois não é sabido se algumas das espécies inventariadas possuem potencial para se tornar praga de cultivares da região.

\section{CONCLUSÃO}

A análise faunística das espécies de gafanhotos Acridoidea da Volta Grande do Rio Xingu evidenciou a existência de um número grande de espécies raras e mínimas, concentradas na área denominada A2 (A2IG, A2IM e A2T50). Tal área, que será inundada para compor o lago de abastecimento da casa de força da usina hidrelétrica de Belo Monte sofrerá um grande impacto ambiental, visto que seu alagamento destruirá os habitats utilizados pelas espécies em geral, inclusive de gafanhotos, o que poderá ocasionar a extinção local destas. Entretanto, são conhecidas as inúmeras relações ecológicas existentes entre os organismos de determinado ambiente e essas relações influenciam de forma direta ou indireta a biota como um todo. Dessa forma, pode-se considerar que a extinção de um número considerável de espécies de gafanhotos, nas áreas da bacia do Rio Xingu, irá comprometer o equilíbrio ambiental da região, visto que esses insetos fazem parte da dieta alimentar de muitas espécies de invertebrados e de vertebrados, que são fundamentais para a manutenção dos ecossistemas. Deve-se, ainda, considerar importante que as espécies aqui tratadas não possuem informações biológicas e nem de sua história natural, o que poderia ser preocupante o fato de a ciência e da humanidade desconhecer o papel e os serviços ecológicos que tais espécies desempenham na Volta Grande do Xingu.

\section{AGRADECIMENTOS}

Os autores agradecem ao Museu Paraense Emílio Goeldi pela oportunidade de participar do Estudo de Impacto Ambiental (EIA) para a implementação da Hidrelétrica de Belo Monte.

\section{REFERÊNCIAS}

AMÉDÉGNATO, C. Diversity in an Amazonian canopy grasshopper community in relation to resource partition and phylogeny. In: STORK, N.E. et al. Canopy Arthropods. London: Chapman and Hall, 1997. p.281-319.

DESCAMPS, M. Étude dês Ecosystemes Guyanais III Acridomorpha Dendrophiles (Orthoptera Caeliphera). Annales de la Societe Entomologique de France, v.14, p.301-349, 1978.

FEARNSIDE, P.M. Hidrelétricas planejadas no Rio Xingu como fontes de gases do efeito estufa: Belo Monte (Kararaô) e Altamira (Babaquara). In: SEVÁ FILHO, A.O. Tenotã-mõ: alertas sobre as consequências dos projetos hidrelétricos no rio Xingu, Pará, Brasil”. São Paulo: International Rivers Network, 2005. p.204-241.

FISHER, W.H. Megadevelopment, environmentalism, and resistance: the institutional context of Kayapó indigenous politics in Central Brazil. Human Organization, v.53, p.220-232, 1994.

FONSECA, G.A.B. et al. Conservation of island biodiversity - importance, challenges and opportunities. Washington, DC: Center for Applied Biodiversity Sciences, Conservation International, 2006. p.16.

GRACIANI, C. et al. Análise faunística de gafanhotos (Orthoptera, Acridoidea) em fragmento florestal próximo ao Rio Uruguai, município de Chapecó, Santa Catarina. Biotemas, v.18, p.87-98, 2005. Disponível em: <https://periodicos.ufsc.br/index. $\mathrm{php} /$ biotemas/article/download/21414/19380>. Acesso em: 03 mar. 2014.

GUERRA, W.D. et al. Gafanhotos (Orthoptera Acridoidea) em áreas de cerrados e lavouras na Chapada dos Parecis, Estado de Mato Grosso, Brasil. Revista Brasileira de Entomologia, v.56, p.228-239, 2012. Disponível em: <http://www.scielo.br/pdf/rbent/ v56n2/aop3012.pdf>. Acesso em: 20 fev. 2014.

LUTINSKI et al. Análise faunística de gafanhotos na Floresta Nacional de Chapecó, Santa Catarina. Pesquisa Florestal Brasileira, v.31, p.43-50, 2011. Disponível em: <http://pfb.cnpf. embrapa.br/pfb/index.php/pfb/article/viewFile/191/202>. Acesso em: 12 mar. 2014. doi: 10.4336/2011.pfb.31.65.43.

LYONS, K.G. et al. Rare species and ecosystem functioning. Conservation Biology, v.19, p.1019-1024, 2005.

MIRANDA, E.E. et al. Efeitos ecológicos das barragens do Xingu: uma avaliação preliminar. In: SANTOS, L.A.; ANDRADE, L.M. As hidrelétricas do Xingu e os povos indígenas. São Paulo: Comissão Pró-Índio, 1988. p.83-102.

NUNES, A.L. et al. Fenologia de Phlugis teres (De Geer, 1927) (Orthoptera-Tettigoniidae) e abundância de artrópodos em populações de Eichhornia crassipes (Pontederiaceae) em um lago de várzea na Amazônia Central. Boletim do Museu Paraense Emílio Goeldi, v.1, p.271-285, 2005.

NUNES-GUTJAHR, A.L.; BRAGA, C.E. Similaridade entre amostras da Acridofauna (Orthoptera: Acrididae) em quatro áreas ao longo da Estrada Santarém-Cuiabá (BR-163), Pará, Brasil. Revista Nordestina de Zoologia, v.4, p.118-130, 2010.

NUNES-GUTJAHR, A.L.; BRAGA, C.E. Gafanhotos In: OLIVEIRA, M.L. et al. Reserva Ducke: a biodiversidade amazônica através de uma grade. Manaus: INPA, 2011. p.131-143.

NUNES-GUTJAHR, A.L.; BRAGA, C.E. Gafanhotos da Flona Caxiuanã: guia prático. Belém: MPEG, 2012. p.62.

RAFAEL, J.A. A amostragem. Protocolo e técnicas de captura de Diptera. In: COSTA, C. et al. Proyecto de red iberoamericana de biogeografia y entomologia sistemática: PRIBES. Zaragosa: Sociedade Entomológica Aragonesa, 2002. v.2, p.301-304.

RODRIGUES, W.C. DivEs - Diversidade de espécies. Versão 2.0. Software e Guia do Usuário, 2005. Disponível em: <http://www. ebras.bio.br>. Acesso em: 17 mar. 2014. 
SERAFINI, T.Z. et al. Ilhas oceânicas brasileiras: biodiversidade conhecida e sua relação com o histórico de uso e ocupação humana. Revista de Gestão Costeira Integrada, v.10, p.281301, 2010. Disponível em: <http://www.aprh.pt/rgci/pdf/rgci178_Serafini.pdf $>$. Acesso em: 15 fev. 2014.

SILVEIRA NETO, S. et al. Manual de ecologia dos insetos. Piracicaba: Agronômica Ceres, 1976. p.419.
SILVEIRA NETO, S. et al. Uso da análise faunística de insetos na avaliação do impacto ambiental. Scientia Agricola, v.52, p.9-15, 1995 .

THOMAZINI, M.J.; THOMAZINI, A.P.B.W. Diversidade de abelhas (Hymenoptera: Apoidea) em inflorescências de Piper hispidinervum (C.D.C.). Neotropical Entomology, v.31, p.27-34, 2002. Disponível em: <http://www.scielo.br/pdf/ne/v31n1/11175. pdf>. Acesso em: 12 fev. 2014. 